Vol. 35 (1), 2017 (ISSN 0254-9247)

\title{
Estrés de rol y empowerment psicológico como antecedentes de la satisfacción laboral
}

\author{
Alejandro Orgambídez-Ramos ${ }^{1}$, Daniel Moura ${ }^{2}$, Helena de Almeida ${ }^{3}$ \\ Universidade do Algarve -Portugal, Research Centre for Spatial \\ and Organizational Dynamics (FCT, Portugal)
}

El objetivo de este estudio fue analizar el impacto del estrés de rol (ambigüedad y conflicto) sobre la satisfacción laboral a través del empowerment psicológico en el trabajo. Se obtuvieron datos de una muestra de 314 trabajadores del sur de Portugal $\left(M_{\text {edad }}=35.59, D E_{\text {edad }}=10.66\right)$. El $69.75 \%$ de los participantes fueron mujeres. Los resultados mostraron que el efecto del conflicto de rol fue directo sobre la satisfacción laboral, mientras que el efecto de la ambigüedad fue mediado por las dimensiones autodeterminación y significado del empowerment psicológico. Las intervenciones en las empresas deben centrarse en la prevención del estrés de rol y en el aumento del empowerment psicológico.

Palabras clave: ambigüedad de rol, conflicto de rol, empowerment psicológico, satisfacción en el trabajo, path analysis.

\section{Role stress and psychological empowerment as antecedents of job satisfaction}

The aim of this study was to examine the impact of role stress (ambiguity and conflict) on job satisfaction through psychological empowerment in the workplace. Data was collected from 314 workers from southern Portugal $\left(\mathrm{M}_{\text {age }}=35.39, \mathrm{SD}_{\text {age }}=10.66 ; 69.75 \%\right.$ women $)$ Results indicated that role conflict had a direct effect on job satisfaction, while the impact of role ambiguity on job satisfaction was totally mediated by meaning and self-determination. Psychosocial interventions in companies should focus on prevention of role stress and promotion of psychological empowerment in the workplace.

Keywords: role ambiguity, role conflict, psychological empowerment, job satisfaction, path analysis.

1 Doctor en Psicología del Trabajo y de las Organizaciones por la Universidad de Huelva, Espańa, y professor investigador en la Faculdade de Ciências Humanas e Sociais da Universidade do Algarve. Dirección postal: Campus de Gambelas 8005-139 Faro. Portugal. Contacto: aoramos@ualg.pt

2 Licenciado en Psicología por la Universidade do Algarve, Portugal, y alumno de Doctorado en Psicología en la Faculdade de Ciências Humanas e Sociais da Universidade do Algarve. Dirección postal: Campus de Gambelas 8005-139 Faro. Portugal. Contacto: a37855@ualg.pt

3 Doctora en Psicología Social y de las Organizaciones por la Universidade do Algarve, Portugal, y docente en la Faculdade de Economia da Universidade do Algarve. Dirección postal: Campus de Gambelas 8005-139 Faro. Portugal. Contacto: halmeida@ualg.pt 


\begin{abstract}
Stresse de papel e empowerment psicológico como antecedentes da satisfaçáo no trabalho

O objetivo deste estudo é analisar o impacte do stresse de papel (ambiguidade e conflito) sobre a satisfação no trabalho através do empowerment psicológico no trabalho. Os dados foram obtidos através de uma amostra de 314 profissionais ativos do sul de Portugal $\left(\mathrm{M}_{\text {idade }}=\right.$ 35.59, $\mathrm{DP}_{\text {idade }}=10.66$ ). 69.75\% dos participantes eram mulheres. Os resultados mostraram que o efeito do conflito de papel sobre a satisfação no trabalho é direto, enquanto que o efeito da ambiguidade sobre a satisfação no trabalho é mediado pelas dimensóes autodeterminação e significado do empowerment psicológico. As intervençôes nas empresas devem centrar-se na prevençáo do stresse de papel e no aumento do empowerment psicológico.

Palavras chave: ambiguidade de papel, conflicto de papel, empowerment psicológico, satisfação no trabalho, path analysis.
\end{abstract}


En la actualidad, el estrés de rol se ha mostrado como uno de los más importantes riesgos psicosociales en el trabajo dado su impacto sobre la salud y el bienestar de las personas, siendo uno de los antecedentes del síndrome de quemarse en el trabajo o burnout (Peiró \& Rodríguez, 2008). La presencia de los roles laborales ayudan a reducir la incerteza en el puesto de trabajo, si bien pueden provocar problemas cuando aparecen el conflicto, la sobrecarga o la ambigüedad de rol (Osca, GonzálezCamino, Bardera \& Peiró, 2003). Consecuentemente, la comprensión de los mecanismos por los que el estrés de rol afecta a la salud y el bienestar de los trabajadores se convierte en un tema de gran relevancia tanto teórica como práctica (Dávila \& Jiménez-García, 2014; EU-OSHA, 2014).

Una de las variables implicadas en la relación entre el estrés de rol y variables asociadas al bienestar del trabajador (i.e., satisfacción laboral) es el empowerment psicológico (EP) en el trabajo. Según Spreitzer, el empowerment psicológico hace referencia a una serie de procesos y estados psicológicos internos de tipo cognitivo (significado, competencia, autodeterminación, impacto), que modifican la percepción del sujeto respecto de sí mismo y del contexto laboral en el que se encuentra (Jáimaz \& Bretones, 2013; Mendoza-Sierra, León-Jariego, Orgambídez-Ramos y Borrego-Alés, 2009; Spreitzer, 1995; Thomas \& Velthouse, 1990). Estas cogniciones representan una orientación o motivación activa hacia el trabajo (Spreitzer, 1995, 1996).

De acuerdo con Spreitzer $(1995,1996)$ y el modelo de empowerment estructural de Laschinger, Finegan, Shamian, y Wil (2001), la falta de información en el trabajo, informaciones contradictorias y/o la ausencia de instrucciones claras sobre el rol profesional afectan negativamente a la experiencia de empowerment psicológico en el trabajo. A su vez, bajos niveles de empowerment en el trabajo se relacionan con bajas puntuaciones en satisfacción laboral y bienestar (Cicolini, Comparcini \& Valentina, 2013; Lautizi, Laschinger \& Ravazzolo, 2009; Orgambídez-Ramos, Borrego-Alés \& Mendoza-Sierra, 2014). 
Si bien existen estudios que analizan las correlaciones entre el estrés de rol, el empowerment psicológico y bienestar en el trabajo, pocos han planteado el análisis específico del papel del empowerment psicológico en la relación entre estrés de rol y satisfacción laboral. En este sentido, dada la necesidad de comprender los procesos asociados al estrés de rol y su influencia en la satisfacción de los trabajadores, el objetivo de esta investigación es analizar el impacto del estrés de rol sobre la satisfacción laboral a través del empowerment psicológico en el trabajo.

\section{Empowerment psicológico en el trabajo}

El concepto de empowerment psicológico está apareciendo en los últimos años como un elemento fuertemente asociado tanto a la salud organizacional como a la eficacia y la calidad de servicio en las empresas (Banda \& Morales-Zamorano, 2015; Mendoza-Sierra et al., 2009; Orgambídez-Ramos et al., 2014). Spreizer (1995), basándose en la propuesta de Thomas y Velthouse (1990), definió el empowerment psicológico como una motivación intrínseca hacia el trabajo, producto de cuatro cogniciones que favorecen una orientación activa hacia el rol laboral: significado, competencia, autodeterminación e impacto.

El significado se refiere a la importancia o valor dado a las metas y objetivos laborales, de acuerdo con los valores y necesidades del trabajador. La competencia se relaciona con la creencia individual en la capacidad para realizar las funciones del puesto con eficacia. La autodeterminación se refiere a la capacidad sentida por el empleado para tomar decisiones relativas a la organización. Finalmente, el impacto se define como el grado en que el trabajador considera que sus acciones afectan a los resultados del departamento, sección u organización (Spreitzer, 1995, 1996). En general, el significado conlleva un alto compromiso y concentración de energía en el trabajo, la competencia se relaciona con el esfuerzo continuado en situaciones laborales difíciles y con el desempeño, la autodeterminación se traduce en interés por el aprendizaje y la perseverancia ante los retos laborales, y el impacto tiene que ver con el desempeño y la constancia. 
Diversos estudios han mostrado que el empowerment psicológico se relaciona con una mayor satisfacción de los empleados, un elevado compromiso con la empresa y una mayor productividad (Cicolini et al., 2013; Seibert, Wang \& Courtright, 2011; Spreitzer, Kizilos \& Nason, 1997; Zhou, Wang, Chen \& Shi, 2012). Relativamente a la relación entre el empowerment psicológico y la satisfacción laboral, se han observado correlaciones positivas y significativas en diversos países y muestras: en personal médico (Cicolini et al., 2013), vendedores de textiles (Erdogan \& Bauer, 2009) o trabajadores de empresas petroquímicas (Yao, 2013). En general, se asume que cuando los trabajadores perciben que tienen autonomía y competencia para realizar tareas con significado y un elevado impacto en la organización, se sienten más satisfechos en sus puestos (Seibert et al., 2011).

\section{Estrés de rol}

En la actualidad, los cambios en el entorno socioeconómico han provocado a su vez el cambio de estructuras y funciones en las empresas para adaptarse a las nuevas exigencias competitivas. Precisamente en situaciones de cambio organizacional, la ambigüedad y el conflicto de rol son dos de las dificultades más frecuentes de los empleados al enfrentarse a las nuevas exigencias y funciones (Osca et al., 2003).

La ambigüedad de rol se define como la falta de información clara relativa a las funciones y responsabilidades asociadas a un puesto (Cervoni \& Delucia-Waack, 2011; Díaz-Funes, Pecino \& Mañas, 2016), es decir, cuando la persona trabaja con incerteza o ausencia de claridad en relación con las tareas que ha de realizar. Esta situación puede ser el resultado de una inadecuada descripción de la función laboral (por ser un puesto de nueva creación, flexible o creativo), de falta de información sobre las metas y procedimientos a seguir o por la incorrecta comunicación de dicha información (Osca et al., 2003). El conflicto aparece cuando se formulan al empleado demandas incompatibles entre sí, de forma que responder a una demanda implica no atender a las otras (Cervoni \& Delucia-Waack, 2011; Díaz-Funes et al., 2016; Osca et al., 2003). 
Diversos estudios han observado que la ambigüedad y el conflicto de rol se correlacionan de forma negativa con actitudes en el trabajo tales como la satisfacción laboral y el compromiso, y de forma positiva con la ansiedad, los trastornos psicosomáticos y el burnout (Cervoni \& Delucia-Waack, 2011; Gil-Monte, Carretero-Dios \& Roldán, 2005; Orgambídez-Ramos et al., 2014; Orgambídez-Ramos, Pérez-Moreno \& Borrego-Alés, 2015). En relación a la satisfacción laboral, dado que el estrés de rol impide a la persona cumplir con sus funciones y lograr sus objetivos por la falta de información y/o pedidos incompatibles, influiría en los sentimientos de placer asociados al trabajo generando evaluaciones negativas del mismo, esto es, bajos niveles de satisfacción laboral.

\section{Relaciones entre estrés de rol, empowerment y satisfacción laboral}

Tanto el modelo de empowerment psicológico de Spreitzer (1995, 1996) como el modelo de empowerment estructural de Laschinger et al. (2001) plantean el papel mediador del empowerment psicológico entre las características del contexto de trabajo y las actitudes laborales, tales como la satisfacción laboral o el compromiso organizacional. De entre los antecedentes señalados por Spreitzer $(1995,1996)$ y Laschinger et al. (2001), se destaca especialmente el acceso a informaciones relativas a los roles profesionales. Sin información, los trabajadores no podrían asumir sus tareas ni tomar decisiones. Igualmente, el estrés de rol (ambigüedad y conflicto) impedirían la adopción efectiva del rol, bloqueando la toma de decisiones, afectando al significado del trabajo que se realiza, impidiendo que las tareas tengan impacto en la organización y alterando las percepciones de maestría o dominio de la tarea. En definitiva, el estrés de rol afectaría negativamente a los niveles de empowerment psicológico percibidos, y el empowerment psicológico reduciría, a su vez, los niveles de satisfacción en el trabajo. 


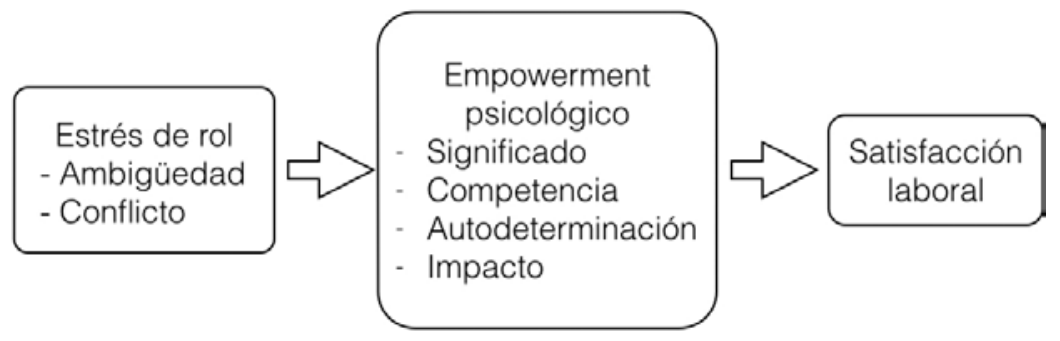

Figura 1. Relaciones entre el estrés de rol, el empowerment psicológico y la satisfacción laboral

Diversos estudios han analizado el papel mediador del empowerment psicológico entre diversos antecedentes, tales como liderazgo (Zhou et al., 2012), exceso de cualificaciones profesionales (Erdogan \& Bauer, 2009), marketing interno (Yao, 2013) o identificación con el trabajo (Butts, Vandenberg, Dejoy, Schaffer \& Wilson, 2009), y la satisfacción laboral. Sin embargo, apenas existen investigaciones que analicen específicamente las relaciones existentes entre el estrés de rol, el empowerment psicológico y la satisfacción laboral planteada en los modelos de Spreitzer (1995, 1996). La comprensión de los mecanismos por los que el estrés de rol afecta a la satisfacción laboral es de gran interés tanto a nivel teórico como a nivel aplicado, ya que permitiría el planteamiento de intervenciones y la gestión de recursos humanos basadas en el empowerment y desde una perspectiva de la prevención y potenciación de los empleados. En consecuencia, el objetivo de este estudio es analizar el impacto del estrés de rol sobre la satisfacción laboral a través del empowerment psicológico en el trabajo. Es decir, se asume el papel mediador del empowerment psicológico entre el estrés de rol (conflicto y ambigüedad) y la satisfacción con el trabajo. De forma específica, se plantean las siguientes hipótesis:

H1: La ambigüedad y el conflicto de rol tienen una relación negativa y significativa con el empowerment psicológico, el cual está positivamente correlacionado con la satisfacción laboral.

H2: El empowerment psicológico media la relación entre la ambigüedad y el conflicto de rol y la satisfacción laboral. 


\section{Método}

\section{Participantes}

Se realizó un estudio ex post facto retrospectivo de un único grupo con múltiples medidas (Montero \& León, 2007), mediante un muestreo por conveniencia en empresas del sur de Portugal desde septiembre a diciembre de 2013. El criterio de selección fue el poseer como mínimo un ańo de experiencia profesional en el puesto de trabajo en el momento de realización de la investigación. Trabajadores autónomos y desempleados no fueron seleccionados.

La muestra estuvo compuesta por 314 participantes, con una media de edad de $35.59(D E=10.66)$ oscilando entre los 18 y los 64 ańos. Del total del muestra, 219 participantes fueron mujeres (69.75\%). En relación al nivel de estudios, el 75.2\% expresó tener estudios superiores (grado o licenciatura). El tiempo medio de permanencia en la empresa se situó en torno a los 7 años $(M=7.02, D E=5.67)$.

\section{Instrumentos}

Empowerment psicológico. Para medir el empowerment psicológico se utilizó la versión portuguesa (Santos, Gonçalves, Orgambídez-Ramos, Mendoza-Sierra \& Borrego-Alés, 2014) de la escala de empowerment psicológico de Spreitzer (1995). La escala está compuesta por 12 ítems repartidos en 4 dimensiones con 3 ítems cada una: significado, competencia, autodeterminación e impacto. Ejemplos de los ítems son los siguientes: "El trabajo que hago es significativo" y "Tengo una gran autonomía para hacer mi trabajo". Los participantes expresan su grado de acuerdo con el enunciado de cada ítem usando una escala tipo Likert que va desde 1: Totalmente en desacuerdo hasta 5: Totalmente de acuerdo. Mayores puntuaciones indican elevados niveles de empowerment psicológico en el lugar de trabajo. La consistencia interna (alpha de Cronbach) obtenida en el estudio fue de .88 para la escala global, y .91, .87, .89 y .92 para significado, competencia, autodeterminación e impacto, respectivamente. 
Estrés de rol. Para evaluar el estrés de rol se usó la versión portuguesa (Almeida, 2009) de la escala de estrés de rol de Rizzo, House y Lirtzman (1970). Los 11 ítems del cuestionario se agrupan en dos dimensiones: ambigüedad de rol (5 ítems) y conflicto de rol (6 ítems). Ejemplos de ítems son los siguientes: "Conozco bien el grado de autonomía en mi trabajo" y "Hago cosas que pueden ser aceptadas por una persona pero no por otra". Los participantes responden al enunciado de cada ítem utilizando una escala tipo Likert, que va desde 1: Muy en desacuerdo a 7: Muy de acuerdo. Elevadas puntuaciones expresan elevados niveles de estrés de rol relativamente a la ambigüedad y el conflicto. A partir de los resultados de los análisis preliminares (ver sección de Resultados), se eliminaron dos ítems del cuestionario de estrés de rol, un ítem de la escala de ambigüedad y un ítem de la escala de conflicto. Los coeficientes de consistencia interna (alpha de Cronbach) obtenidos fueron de .80 para ambigüedad de rol y .79 para conflicto de rol.

Satisfacción en el trabajo. Para medir la satisfacción en el trabajo se aplicó la escala de satisfacción laboral de Lima, Vala y Monteiro (1994). Esta escala consta de 8 ítems que se responden utilizando una escala Likert que va desde 1: Totalmente insatisfecho hasta 7: Totalmente satisfecho. Ejemplos de ítems son los siguientes: "En relación a sus oportunidades de promoción, diría que está..." y "En relación al trabajo que realiza, diría que está...”. A partir de los resultados de los análisis preliminares (ver sección de Resultados), se eliminó uno de los ítems de la escala de satisfacción laboral por su baja carga factorial. El coeficiente alpha de Cronbach obtenido en este estudio fue de .83 .

\section{Procedimiento}

La recogida de datos se realizó mediante cuestionarios de lápiz y papel aplicados individualmente y en horario laboral. Los investigadores se desplazaron por las varias empresas de la región y, tras reunirse con la dirección de la empresa, solicitaban la participación de la misma en la investigación. Tras la autorización de la dirección 
de la organización, los cuestionarios fueron distibuidos a todos los trabajadores y empleados que voluntariamente se ofrecieron a participar, los cuales dieron su consentimiento verbalmente. Antes de iniciar la aplicación de las escalas, las instrucciones del cuestionario fueron explicadas, asegurándose la total confidencialidad y anonimato de las informaciones aportadas.

\section{Análisis de datos}

Para la comprobación de las hipótesis, se ha usado el paquete estadístico STATA v.13. Se han realizado análisis descriptivos (media, desviación típica, asimetría), se han calculado las consistencias internas (alpha de Cronbach) y se han calculado las correlaciones (coeficientes de Pearson) entre ellas.

El sistema de relaciones e hipótesis asociadas a la Figura 1 se puso a prueba a través un sistema de ecuaciones estructurales basado en correlaciones y tratando a las variables como observables (path analysis). El método de estimación de parámetros utilizado fue el de máxima verosimilitud (Maximum Likelihood). Las variables que se relacionan en el modelo son puntuaciones en tests, siendo tratadas como medidas falibles que contienen error de medida (Acock, 2013). El análisis de mediación se realizó siguiendo las recomendaciones de Hayes (2013) y se utilizó el test de Sobel (1992) para el cálculo de los efectos directos e indirectos.

\section{Resultados}

\section{Análisis preliminares}

Modelo de medida. Se llevó a cabo un análisis factorial confirmatorio para comprobar la validez discriminante de los constructos del estudio (Tabla 1). En primer lugar, se puso a prueba un modelo (M1a) con una estructura de siete factores: un factor de ambigüedad de rol, un factor de conflicto de rol, cuatro factores de empowerment psicológico (significado, impacto, autodeterminación, competencia) y un factor 
de satisfacción laboral. Este modelo mostró un ajuste insatisfactorio, donde dos ítems de la escala de estrés de rol (ítems 1 y 6) y un ítem de la escala de satisfacción laboral (ítem 6) mostraron bajas saturaciones $(<.30)$. Tras la eliminación de estos tres ítems, se puso a prueba un nuevo modelo (M1b) que logró mejores índices de ajuste de acuerdo con las recomendaciones de Hu y Bentler (1999), si bien el índice TLI (Tucker-Lewis Index) lo llegó al valor mínimo de ajuste de .90. No obstante, el resto de los indicadores sugieren que las variables pueden ser consideradas como constructos independientes.

\section{Tabla 1}

Indices de ajuste de los modelos probados

\begin{tabular}{cccccccc}
\hline & $X^{2}$ & $G l$ & CFI & TLI & AIC & RMSEA & SRMR \\
\hline M1a & $896.537^{* *}$ & 413 & .890 & .877 & 44838.551 & .066 & .074 \\
M1b & $757.025^{* *}$ & 329 & .901 & .887 & 39354.533 & .069 & .069 \\
M1c & $3175.77^{* *}$ & 377 & .360 & .311 & 43373.001 & .170 & .140 \\
M2 & $41.42^{* *}$ & 4 & .817 & .588 & 34499.960 & .185 & .074 \\
M3 & 3.128 & 3 & .999 & .998 & 3413.168 & .013 & .02 \\
\hline
\end{tabular}

Nota. ${ }^{*} \mathrm{p}<.05 ;{ }^{* *} \mathrm{p}<.01 . X^{2}$ (chi-cuadrado); $g l$ (grados de libertad); CFI (Comparative Fit Index); $T L I$ (Tucker-Lewis Index); AIC (Akaike Information Criterion); RMSEA (Root Mean Square Error of Aproximation); SRMR (Standardized Root Mean Square Residual)

Varianza común del método. En siguiente lugar, dado que todos los datos están basados en autoinformes y fueron recogidos en el mismo periodo de tiempo, la varianza común asociada al método (Common Method Variance) puede sobreestimar o infravalorar las relaciones entre variables (Podsakoff, MacKenzie \& Podsakoff, 2012). Para examinar el posible efecto de la varianza común, se puso a prueba un tercer modelo (M1c) donde todos los ítems de las escalas del estudio saturaban en solo un factor. El ajuste del modelo M1c fue insatisfactorio (Tabla 1). En consecuencia, aunque no puede excluirse totalmente el efecto de la varianza común, no parece afectar de forma significativa a las relaciones entre las variables estudiadas. 


\section{Descriptivos y correlaciones}

La Tabla 2 muestra los estadísticos descriptivos - medias, desviaciones típicas, asimetría - y correlaciones de las variables del estudio, junto con la fiabilidad de las escalas. Tanto la ambigüedad como el conflicto de rol registraron valores inferiores a la media de la escala, siendo el valor más bajo el correspondiente a la ambigüedad de rol. Relativamente a las dimensiones del empowerment psicológico, sus valores medios se situaron por encima de la media de la escala, con el valor más elevado el correspondiente a significado y el más bajo a impacto. Finalmente, la satisfacción laboral se situó por encima del valor medio de la escala.

\section{Tabla 2}

Estadisticos descriptivos y correlaciones de las variables del estudio

\begin{tabular}{lccccccc}
\hline & 1 & 2 & 3 & 4 & 5 & 6 & 7 \\
\hline 1. Ambigüedad & $(.80)$ & & & & & & \\
2. Conflicto & $.24^{* *}$ & $(.79)$ & & & & & \\
3. Significado & $-.41^{* *}$ & -.08 & $(.91)$ & & & & \\
4. Competencia & $-.50^{* *}$ & -.07 & $.48^{* *}$ & $(.87)$ & & & \\
5. Autodeterminación & $-.31^{* *}$ & -.06 & $.45^{* *}$ & $.32^{* *}$ & $(.89)$ & & \\
6. Impacto & $-.24^{*}$ & -.06 & $.25^{* *}$ & $.34^{* *}$ & $.42^{* *}$ & $(.92)$ & \\
7. Satisfacción & $-.31^{* *}$ & $-.39^{* *}$ & $.34^{* *}$ & $.18^{* *}$ & $.32^{* *}$ & $.20^{* *}$ & $(.83)$ \\
Media & 2.31 & 3.27 & 4.27 & 4.24 & 3.82 & 3.19 & 4.36 \\
Desviación Típica & .92 & 1.58 & .71 & .58 & .91 & 1.21 & 1.18 \\
Asimetría & .73 & .02 & -1.40 & -1.01 & -1.09 & -.61 & -.32 \\
\hline
\end{tabular}

Nota. ${ }^{* *} p<.01$. Los coeficientes alpha de Cronbach se muestran en la diagonal.

Los resultados del análisis de correlación muestran que, tal y como se esperaba, los estresores de rol se correlacionaron negativamente con la satisfacción laboral, con valores de Pearson de $-.31(p<.01)$ para ambigüedad de rol y de -.39 $(p<.01)$ para conflicto de rol. Por el contrario, todas las dimensiones del empowerment psicológico obtuvieron correlaciones significativas $(p<.01)$ y positivas con la satisfacción, con valores de $.34, .18, .32$ y .20 para significado, competencia, autodeterminación e impacto, respectivamente. 


\section{Análisis de mediación}

Con el objetivo de lograr una representación global de las relaciones entre estrés de rol, empowerment psicológico y satisfacción laboral, se ha diseńado un modelo de relaciones donde la ambigüedad y el conflicto de rol son variables exógenas y predictoras, el empowerment psicológico es la variable endógena y mediadora, y la satisfacción laboral es la variable endógena y resultado. El modelo ha sido comprobado empíricamente a partir del análisis de ecuaciones estructurales, utilizando el método path analysis con máxima verosimilitud como procedimiento de estimación de parámetros. De acuerdo con las directrices de Acock (2013), el análisis se ha realizado siguiendo dos pasos: (I) diseño de un modelo sobre-identificado (M2) y (II) rediseño del modelo (M3) a partir de los coeficientes significativos observados en el modelo anterior. Los índices de ajuste de los modelos M2 y M3 pueden consultarse en la Tabla 1.

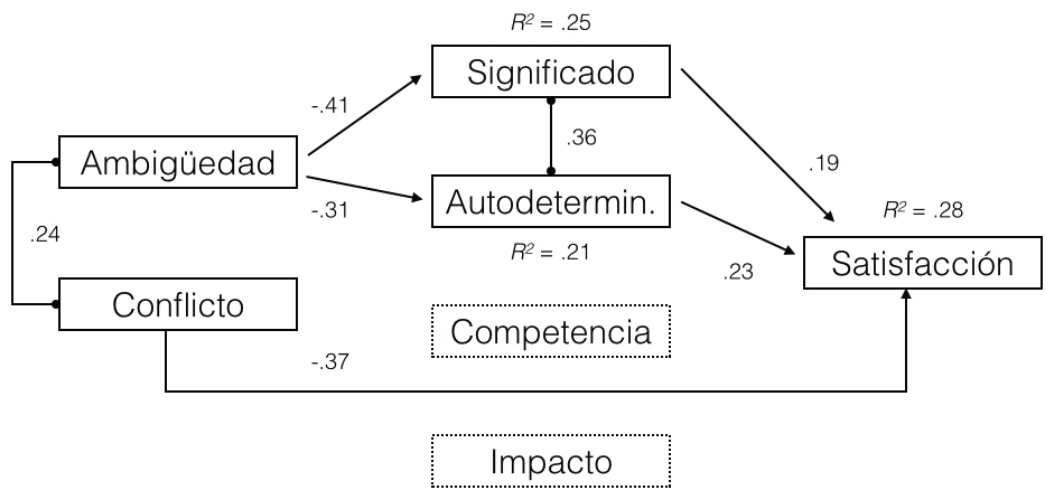

Nota: Todos los coeficientes son estadísticamente significativos $(p<.01)$

Figura 2. Resultados del path analysis. Coeficientes estandarizados y valores de $R^{2}$ de las variables endógenas $(N=314)$

La Figura 2 presenta los coeficientes estandarizados y los niveles de significatividad del modelo final (M3), así como los valores de la varianza explicada $\left(R^{2}\right)$ de las dimensiones significado y autodeterminación del empowerment psicológico, y de la satisfacción laboral. 
El ajuste del modelo M3 fue adecuado: $X^{2}(3)=3.128, p=.372$, con los valores CFI (Comparative Fit Index) y TLI (Tucker-Lewis Index) superiores a .95 (.999 y .998, respectivamente) y los valores SRMR (Standardized Root Mean Square Residual) y RMSEA (Root Mean Square Error of Approximation) inferiores a .05 (.021 y .013, respectivamente).

Se puede observar que solo la ambigüedad de rol predijo de forma negativa y significativa $(p<.01)$ las dimensiones significado $(-.41)$ y autodeterminación (-.31) del empowerment psicológico, explicando un 10\% y un $17 \%$ de la varianza de ambas dimensiones, respectivamente. En relación a la satisfacción laboral, tanto el conflicto de rol (-.37) como autodeterminación (.19) y significado (.23) se mostraron como predictores significativos $(p<.01)$, dando cuenta de un $28 \%$ del total de su varianza.

Relativamente al papel mediador del empowerment psicológico en el trabajo entre el estrés de rol y la satisfacción laboral, las dimensiones autodeterminación y significado mediaron totalmente el impacto de la ambigüedad de rol sobre la satisfacción, $z=-5.22, p<.01$. En el caso del conflicto de rol, su impacto sobre la satisfacción laboral fue directo $(-.37, p<.01)$, sin existir cualquier tipo de mediación a través del empowerment psicológico. En la Tabla 3 se muestran los efectos directos e indirectos sobre la satisfacción laboral.

\section{Tabla 3}

Coeficientes estandarizados de los efectos directos, indirectos y totales de estrés de rol y empowerment sobre satisfacción laboral

\begin{tabular}{lcccc}
\hline \multicolumn{1}{c}{ Resultado } & Efecto directo & Efecto indirecto & $z$ & Efecto total \\
\hline $\begin{array}{l}\text { Significado } \\
\text { Ambigüedad }\end{array}$ & -.41 & - & -.41 \\
$\begin{array}{l}\text { Autodeterminación } \\
\text { Ambigüedad }\end{array}$ & -.31 & - & .31 \\
Satisfacción & & & \\
Significado & $.19^{* *}$ & - & \\
Autodeterminación & $.23^{* *}$ & - & & .19 \\
Ambigüedad & - & -.16 & 5.22 & -.16 \\
Conflicto & -.37 & - & & -.37 \\
\hline
\end{tabular}

Nota: Todos los coeficientes fueron significativos $(p<.01)$ 
Estrés de rol y empowerment psicológico como antecedentes... / Orgambídez-Ramos et al.

\section{Discusión}

Los estresores de rol están apareciendo en los contextos organizacionales con mayor fuerza dadas las nuevas exigencias competitivas y las transformaciones en las empresas. El estudio y la comprensión de los procesos asociados al estrés de rol se revela fundamental por sus repercusiones para la salud laboral y la eficacia y la productividad (Gil-Monte \& Neveu, 2013; Peiró \& Rodríguez, 2008). En este sentido, el objetivo de este estudio ha sido analizar el impacto del estrés de rol sobre la satisfacción laboral a través de la percepción de empowerment psicológico en el trabajo. Los resultados mostraron que la influencia de la ambigüedad de rol sobre la satisfacción resultó mediada por las dimensiones autodeterminación y significado del empowerment, mientras que el efecto del conflicto de rol sobre la satisfacción fue directo.

Sobre la relación entre EP y satisfacción laboral, las dimensiones significado y autodeterminación se mostraron como predictores positivos y significativos. Estos resultados están en línea con los observados en otras investigaciones (Cicolini et al., 2013; Lautizi et al., 2009; Orgambídez-Ramos et al., 2014; Spreitzer et al., 1997; Yao, 2013). Spreitzer et al. (1997), con dos muestras de empleados norteamericanos, una de supervisores y otra de empleados de base, observaron igualmente que el significado y la autodeterminación fueron predictores significativos de la satisfacción, mientras que la competencia y el impacto de relacionaron más con la eficacia y el desempeño en el trabajo. En los estudios de Orgambídez-Ramos et al. (2014) y Lautizi et al. (2009), el empowerment fue predictor significativo y positivo de la satisfacción laboral en muestras de docentes y de personal de enfermería. En la investigación de Yao (2013), el significado también fue la dimensión del EP con mayor peso sobre la satisfacción laboral.

Trabajos con apenas significado parecen estar asociados a sentimientos de apatía y de insatisfacción laboral. Por otro lado, la autodeterminación es uno de los elemento clave de la motivación intrínseca, antecedente de la satisfacción. Mayores niveles de autonomía y la capacidad para tomar decisiones se asocian a valoraciones 
más positivas del trabajo, aumentando las percepciones de satisfacción laboral (Laschinger, Finegan, Shamian \& Wilk, 2004; Spreitzer et al., 1997; Seibert et al., 2011).

Relativamente al modelo planteado ente estrés de rol, empowerment y satisfacción laboral, el conflicto de rol tuvo un impacto directo sobre la satisfacción laboral mientras que la ambigüedad de rol fue totalmente mediada por las dimensiones significado y autodeterminación del empowerment psicológico. El conflicto de rol ocurre cuando aparecen demandas incompatibles o incoherentes en un puesto de trabajo, de forma que atender a una demanda conlleva no responder a las otras (Cervoni \& Delucia-Waack, 2011). Los conflictos entre demandas incompatibles influenciarían negativamente los estados mentales positivos y a las actitudes frente al trabajo. Dado que el conflicto de rol surge de la interacción con los demás (colegas, supervisores, subordinados) y no de las características de la tarea en sí, posee cierto grado de incontrolabilidad que aumenta los niveles de estrés y de insatisfacción experimentados en el trabajo (Orgambídez-Ramos et al., 2015).

Frente al impacto directo del conflicto de rol sobre la satisfacción laboral, la ambigüedad de rol fue totalmente mediada por las dimensiones significado e autodeterminación del EP. La ambigüedad de rol ocurre cuando una persona no sabe cuáles son sus tareas y responsabilidades en un puesto dentro de una empresa (Cervoni \& Delucia-Waack, 2011; Osca et al., 2003). De acuerdo con la teoría de rol (Rizzo et al., 1970), cada posición en la organización debe tener claramente definidas sus tareas y responsabilidades. Si los trabajadores no conocen su grado de autoridad/autonomía y sus tareas y responsabilidades, estos vacilarán cuando deban tomar decisiones (falta de autodeterminación) sintiéndose menos motivados y satisfechos en el trabajo. Por otro lado, solo cuando las personas entienden su papel en la organización, perciben que su trabajo tiene sentido y significado en el global de la empresa, aumentando sus percepciones de significado y sus niveles de satisfacción laboral (Cicolini et al., 2013; Seibert et al., 2011; Spreitzer et al., 1997).

La naturaleza propia de cada estresor de rol ayuda a entender la razón por la que la ambigüedad resulta mediada y el conflicto no. 
El conflicto de rol está asociado a las expectativas contradictorias entre diferentes personas, es decir, está más relacionada con las interacciones con los demás. En cambio, la ambigüedad de rol se refiere a las informaciones necesarias para realizar tareas en el puesto de trabajo, de forma que afecta a las cogniciones del empowerment psicológico. Por ello, el conflicto de rol tiene un impacto directo sobre la satisfacción - con los compañeros, con los jefes_ mientras que la ambigüedad es mediada por el empowerment psicológico. La falta de información, de claridad, impide la toma de decisiones por parte de los empleados (autodeterminación) de forma que el trabajo y las tareas no tienen sentido (significado), afectando negativamente a la satisfacción laboral.

No obstante, los resultados obtenidos en este estudio han de interpretarse considerando ciertos aspectos. En primer lugar, el diseńo transversal utilizado no permite establecer relaciones de causalidad entre las variables (León \& Montero, 2003), aunque esta investigación plantea relaciones de este tipo en el modelo. No obstante, los modelos teóricos de Spreitzer (1995) y de Laschinger et al. (2001) permiten plantear estas relaciones entre las variables estudiadas.

Otro aspecto a tener en cuenta es la medición de las variables del estudio a través de medidas de autoinforme. Este tipo de medición aumenta el riesgo de varianza del método común (Podsakoff et al., 2012). Si bien el resultado del análisis factorial confirmatorio indica que este tipo de varianza no parece afectar especialmente, no puede garantizarse totalmente su falta de influencia en las relaciones estudiadas. Por último, el diseńo de encuestas es muy sensible a ciertos sesgos de respuestas, como el sesgo de deseabilidad social o el sesgo en las respuestas de aquellas personas que voluntariamente se ofrecieron a participar en el estudio (Navas, 2002).

Los resultados observados indican que el conflicto de rol es un predictor directo y negativo de la satisfacción laboral, mientras que el efecto de la ambigüedad de rol es mediado por las dimensiones significado y autodeterminación del empowerment psicológico. Consecuentemente, las políticas de recursos humanos y las intervenciones psicosociales en las organizaciones deben orientarse tanto a la prevención de este 
tipo de estresor psicosocial como en el aumento del empowerment psicológico, utilizando prácticas de recursos humanos que favorezcan la claridad de rol, la autonomía y la toma de decisiones y la realización de un trabajo con sentido y significado. Igualmente, la creación de redes de apoyo y una comunicación fluida y transparente son elementos a implementar en las organizaciones por su impacto en el bienestar de los trabajadores.

Finalmente, futuras investigaciones deben orientarse hacia la evaluación del impacto diferencial del estrés de rol y del empowerment psicológico en la satisfacción de los trabajadores (con las características del trabajo, con los supervisores, con los compañeros). Igualmente, las investigaciones deberán tener un carácter longitudinal y ser realizadas con diferentes muestras de profesionales y trabajos. Por último, la inclusión de nuevas variables, como apoyo organizacional, facilitaría un mejor entendimiento de los procesos psicológicos y psicosociales asociados a los estresores de rol y sus consecuencias sobre el bienestar.

\section{Referencias}

Acock, A.C. (2013). Discovering Structural Equation Modeling Using Stata. Texas, US: StataCorp LP.

Almeida, S. C. (2009). O Comprometimento Organizacional e o Stress de Papel na Força de Vendas. Dissertaçáo de Mestrado. Universidade do Porto, Oporto (Portugal).

Banda, A.L. \& Morales-Zamorano, M.A. (2015). Empoderamiento psicológico: Un modelo de sintético con componentes individuales y comunitarios. Revista de Psicología, 33(1), 3-20.

Butts, M.M., Vandenberg, R.J., Dejoy, D.M., Schaffer, B.S. \& Wilson, M. G. (2009). Individual reactions to high involvement work processes: Investigating the role of empowerment and perceived organizational support. Journal of Occupational Health Psychology, 14(2), 122-136. https://doi.org/10.1037/a0014114 
Cervoni, A.M. \& Delucia-Waack, J. (2011). Role conflict and ambiguity as predictors of job satisfaction in high school counselors. Journal of School Counseling, 9(1), 1-30.

Cicolini, G., Comparcini, D. \& Valentina, S. (2013). Workplace empowerment and nurses' job satisfaction: A systematic literature review. Journal of Nursing Management, 22(7), 855871. https://doi.org/10.1111/jonm.12028

Dávila, C. \& Jiménez-García, G. (2014). Sentido de pertenencia y compromiso organizacional: Predicción del bienestar. Revista de Psicología, 32(2), 271-302.

Díaz-Funez, P.A., Pecino, V. \& Mañas, M.A. (2016). Ambigüedad de rol, satisfacción laboral y ciudadanía orgaizacional en el sector público: Un estudio de mediación multinivel. Revista de Psicología, 34(2), 387-412. https://doi.org/10.18800/ psico.201602.007

Erdogan, B. \& Bauer, T. N. (2009). Perceived overqualification and its outcome: The moderating role of empowerment. Journal of Applied Psychology, 94(2), 557-565. https://doi.org/10.1037/ a0013528

EU-OSHA. (2014). Healthy workers: Manage stress. Luxemburg: European Agency of Safety and Health for Work.

Gil-Monte, P. R., Carretero-Dios, H. \& Roldán, M. D. (2005). Algunos procesos psicosociales sobre el síndrome de quemarse por el trabajo (burnout) en profesionales de enfermería. Ansiedad y Estrés, 11(2-3), 281-291.

Gil-Monte, P. R. \& Neveu, J. P. (2013). El síndrome de quemarse por el trabajo (burnout). En B. Moreno-Jiménez y E. Garrosa (Eds.), Salud laboral (pp. 155-177). Madrid: Pirámide.

Hayes, A.F. (2013). Introduction to mediation, moderation, and conditional process analysis. A regression-based approach. London: The Guilford Press.

Hu, L. \& Bentler, P. M. (1999). Cutoff criteria for fit indexes in covariance structure analysis: Conventional criteria versus new alternatives. 
Structural Equation Modeling: A Multidisciplinary Journal, 6(1), 1-55. https://doi.org/10.1080/10705519909540118

Jáimaz, M.J. \& Bretones, F.D. (2013). Spanish adaptation of the structural empowerment scale. The Spanish Journal of Psychology, 16(E4), 1-7. https://doi.org/10.1017/sjp.2013.14

Laschinger, H.K.S., Finegan, J., Shamian, J. \& Wilk, P. (2001). Impact of structural and psychological empowerment on job strain in nursing work settings: Expanding Kanter's model. Journal of Nursing Administration, 31(5), 260-272. https://doi. org/10.1097/00005110-200105000-00006

Laschinger, H. K. S., Finegan, J., Shamian, J. \& Wilk, P. (2004). A longitudinal analysis of the impact of workplace empowerment on work satisfaction. Journal of Organizational Behavior, 25(4), 527-545. https://doi.org/10.1002/job.256

Lautizi, M., Laschinger, H. K. S. \& Ravazzolo, S. (2009). Workplace empowerment, job satisfaction, and job stress among Italian mental health nurses: An exploratory study. Journal of Nursing Management, 17(4), 446-452. https://doi.org/10.1111/j.13652834.2009.00984.x

León, O. G. \& Montero, I. (2003). Métodos de investigación en psicología y educación ( $3^{\mathrm{a}} \mathrm{ed}$.). Madrid: McGrawHill.

Lima, M., Vala, J. \& Monteiro, M. B. (1994). Culturas organizacionais. En M. B. Vala, M. B. Monteiro y A. Caetano (Eds.), Psicologia social e das organizaçôes - Estudos em empresas portuguesas. Lisboa: Celta Editora.

Mendoza-Sierra, M. I., León-Jariego, J.C., Orgambídez-Ramos, A. \& Borrego-Alés, Y. (2009). Evidencias de validez de la adaptación española de la organizational empowerment scale. Revista de Psicologia del Trabajo y de las Organizaciones, 25(1), 17-28. https:// doi.org/10.4321/S1576-59622009000100003

Montero, I. \& León, O. G. (2007). A guide for naming research studies in Psychology. International Journal of Clinical and Health Psychology, 7, 847-862. 
Navas, J. M. (2002). Métodos, diseños y técnicas de investigación psicológica. Madrid: UNED.

Orgambídez-Ramos, A., Borrego-Alés, Y. \& Mendoza-Sierra, M.I. (2014). Role stress and work engagement as antecedents of job satisfaction in Spanish workers. Journal of Industrial Engineering and Managament, 7(1), 360-372. https://doi.org/10.3926/ jiem. 992

Orgambídez-Ramos, A., Pérez-Moreno, P.J. \& Borrego-Alés, Y. (2015). Estrés de rol y satisfacción laboral: Examinando el papel mediador del engagement. Journal of Work and Organizational Psychology, 31(2), 71-77. doi: 10.1016/j.rpto.2015.04.001

Osca, A., González-Camino, G., Bardera, P. \& Peiró, J.M. (2003). Estrés de rol y su influencia sobre el bienestar psíquico y físico en soldados profesionales. Psicothema, 15(1), 54-57.

Peiró, J. M. \& Rodríguez, I. (2008). Estrés laboral, liderazgo y salud organizacional. Papeles del Psicólogo, 29(1), 68-82.

Podsakoff, P.M., MacKenzie, S. \& Podsakoff, N.P. (2012). Source of method bias in social science research and recommendations on how to control it. Annual Review of Psychology, 63, 539-569. https://doi.org/10.1146/annurev-psych-120710-100452

Rizzo, J. R., House, R. J. \& Lirztman, S. I. (1970). Role conflict and ambiguity in complex organizations. Administrative Science Quaterly, 15, 150-163. https://doi.org/10.2307/2391486

Santos, J., Gonçalves, G., Orgambídez-Ramos, A., Mendoza-Sierra, M. I. \& Borrego-Alés, Y. (2014). Estudo de adaptação da escala de empowerment psicológico de Spreitzer (1995) numa amostra portuguesa. Avaliação Psicológica, 13(3), 56-61.

Seibert, S. E., Wang, G. \& Courtright, S.H. (2011). Antecedents and consequences of psychological and team empowerment in organizations: A meta-analytic review. Journal of Applied Psychology, 96(5), 981-1003. https://doi.org/10.1037/a0022676

Sobel, M.E. (1992). A symptotic confidence intervals for indirect effects in structural equation models. En S. Leinhardt (Ed.), 
Sociological Methodology (pp. 290-312). Washington, DC: American Sociological Association.

Spreitzer, G.M. (1995). Psychological empowerment in the workplace: Dimensions, measurement, and validation. Academy of Managament Journal, 38(5), 1442-1465. https://doi. org/10.2307/256865

Spreitzer, G. M. (1996). Social structural characteristics of psychological empowerment. The Academy of Management Journal, 39, 483504. https://doi.org/10.2307/256789

Spreitzer, G.M., Kizilos, M. \& Nason, S. (1997). A dimensional analysis of the relationship between psychological empowerment and effectivenes, satisfaction and strain. Journal of Management, 23(5), 69-704. https://doi.org/10.1177/014920639702300504

Thomas, K.W. \& Velthouse, B.A. (1990). Cognitive elements of empowerment: An "interpretive" model of intrinsic task motivation. Academy of Management Review, 15(4), 666-681. https://doi.org/10.5465/amr.1990.4310926

Yao, Q. (2013). How internal marketing can cultivate psychological empowerment and enhance employee performance. Social Behavior and Personality, 41(4), 529-538. https://doi. org/10.2224/sbp.2013.41.4.529

Zhou, L., Wang, M., Chen, G. \& Shi, J. (2012). Supervisors' upward exchange relationships and subordinate outcomes: Testing the multilevel mediation role of empowerment. Journal of Applied Psychology, 97(3), 668-680. https://doi.org/10.1037/a0026305

Recibido: 02 de febrero, 2016 Revisado: 27 de septiembre, 2016 Aceptado: 14 de octubre, 2016 